Research Journal of Biological Sciences 14 (3-6): 61-65, 2019

ISSN: $1815-8846$

(C) Medwell Journals, 2019

\title{
Evaluating the Effect of Vitamin D and Calcium on Glucose and Insulin Level of Induced-Diabetic Rats
}

\author{
Fatima Salem and Ahmed Abu-Zaiton \\ Department of Biological Sciences, Al-Albayt University, Mafraq, Jordan
}

\begin{abstract}
Diabetes Mellitus (DM) is a major health concern all over the world. The number of affected people is increasing every year. Many factors contribute to the disease including diet, genetics, lifestyle and so forth. The disease is characterized by hyperglycemia because of insuffiency of the insulin producing beta cells of the pancreas. There is need for alternatives to the conventional antidiabetic drugs to overcome their shortages vitamin D and calcium intake are thought to protect against DM our research aims to evaluate the effect of administration vitamin $\mathrm{D}$, calcium and combination of both on glucose and insulin levels in diabetic rats. Diabetes mellitus was induced intraperitoneally in male Wister albino rats by injection of alloxan $\left(150 \mathrm{mgkg}^{-1}\right.$ bw). Animals were divided into seven groups of eight rats each: group I served as normal control, group II act as diabetic control. The diabetic rats treated groups (III, IV, V, VI and VII) were treated, respectively with metformin at dose of $100 \mathrm{mgkg}^{-1}$, vitamin $\mathrm{D}_{3}$ at dose of $12.5 \mathrm{\mu gkg}^{-1}$, calcium gluconate at dose of $100 \mathrm{mgkg}^{-1}$ both vitamin $\mathrm{D}_{3}$ and calcium gluconate and olive oil at dose of $0.3 \mathrm{mLkg}^{-1}$ (serve as vehicle group). The treatments were performed by oral administration once a day for 28 consecutive days. The results showed that vitamin $\mathrm{D}_{3}$ and vitamin $\mathrm{D}_{3}$-calcium combination lowers blood glucose level and calcium alone was not statistically significant decreased glucose level. Supplementation with vitamin $\mathrm{D}_{3}$ with or without calcium increases insulin slightly when compared with normal control.
\end{abstract}

Key words: Vitamin D, Alloxan, Diabetic agent, pancreatic cells, lifestyle. parathyroid glands

\section{INTRODUCTION}

Diabetes Mellitus (DM) is a chronic disorder of carbohydrates metabolism resulting to increase the blood glucose levels, caused by deficiency in insulin secretion or insulin resistance (Bastaki, 2005; Afifi and Kasabri, 2013).

The numbers of diabetic patients has increased in many countries of the world the estimated worldwide prevalence was $2.8 \%$ in 2000 and is predicted to be $4.4 \%$ in 2030. Thus, the prevention of DM is of much greater importance than in 20th century. Focusing on the role of dietary factors and life style in the development of diabetes pmay play important role to preventing it. Based on basic and animal studies, vitamin D and calcium have been suspected as modifiers of diabetes risk (Kumar et al., 2012; Noda and Mizoue, 2010).

Vitamin $\mathrm{D}$ is a lipid-soluble consist from cholecalciferol (vitamin $\mathrm{D}_{3}$ ) ergocalciferol (vitamin $\mathrm{D}_{2}$ ) (Holick and Chen, 2008). Peoples are getting the vitamin D by exposure of skin to UVB, supplements and diet (Holick and Chen, 2008; Kochupillai, 2008). Calcium is the most important physiological cation in the body and it is responsible for many skeletal functions within the body and it is essential component for insulin secretion as it is a calcium-dependent process (Pravina et al., 2013).
Recent studies recorded that changes in vitamin D and calcium homeostasis may cause diabetes. Deficiency of vitamin D cause reducing insulin secretion and insulin resistance which lead to decreasing inflammatory protein factors that play important role in pancreatic $\beta$-cell apoptosis (Holick and Chen, 2008; Mathieu and Gysemans, 2006).

Increasing the calcium intake inversely correlated with body mass and adiposity, adequate calcium consumption could indirectly cause an improvement in vitamin D homeostasis which explains the potential role of calcium in development of diabetes. Level of serum $25(\mathrm{OH}) \mathrm{D}$ is correlated with calcium intake because the half-life of 25(OH)D was longer in case of high calcium intake leading to activate of insulin secretion (Lips, 2012).

Accordingly, recent studies have recorded a relationship between a dietary vitamin $\mathrm{D}$, calcium intake and a reduced risk of subsequent diabetes mellitus. These results have led to the sight that amelioration of glucose level could be achieved by an management in vitamin D and calcium status in diabetic people. Evaluating the effects of vitamin D injection with or without calcium on sugar levels have been inconsistent (Nikooyeh et al., 2011). The aim of this research was to study the effects of vitamin $\mathrm{D}$ and calcium supplementation alone or in combination on the glycemic status, insulin concentration and pancreatic enzymes concentration.

Correspinding Author: Ahmed Abu-Zaiton, Department of Biological Sciences, Al-Albayt University, Mafraq, Jordan 
Res. J. Biol. Sci., 14 (3-6): 61-65, 2019

\section{MATERIALS AND METHODS}

Animals: Adult male Wistar albino rats (220-270 g) were obtained from the animal house of the Faculty of Veterinary Medicine, Jordan University of Science and Technology. Rats were housed under conventional conditions of temperature. They were fed standard dry pellet diet and water ad libitum. methods described were reviewed and processed by Animal Ethics Committee of Jordan University of Science and Technology.

Diabetes induction: Diabetes was induced intraperitoneally injection in rats of alloxan monohydrate (Sigma-Aldrich, St. Louis, MO, USA) at a dose of $150 \mathrm{mgkg}^{-1}$ bw (Suganya et al., 2012). Diabetes was confirmed by measuring of blood glucose concentration using One Touch Basic Glucometer. Animals with a blood glucose level above $200 \mathrm{mgdL}^{-1}$ were selected for the studying.

Experimental design: The animals were randomly divided into experimental groups and control $(\mathrm{n}=8)$. Application of treatments was performed to experimental groups once a day for 28 consecutive days via. orally administration by gavage. Animals groups were assigned as follow.

Group I: Non-diabetic rats left without treatment as normal control.

Group II: Diabetic rats left without treatments as negative control.

Group III: Diabetic rats were treated with metformin dissolved in distilled water at a dose of $100 \mathrm{mgkg}^{-1} \mathrm{bw}$ (Pournaghi et al., 2012) as positive control.

Group IV: Diabetic rats were submitted to supplementation with vitamin $\mathrm{D}_{3}$ (Cholecalciferol) (Sigma-Aldrich, St. Louis, MO,USA) dissolved in $0.3 \mathrm{~mL}$ olive oil at a dose of $12.5 \mu \mathrm{gkg}^{-1} \mathrm{bw}$ (Santos and Vianna, 2005).

Group V: Diabetic rats were submitted to supplementation with calcium gluconate (Acros Organic, New Jersey, USA; Purity 99\%) dissolved in distilled water at a dose of $100 \mathrm{mgkg}^{-1} \mathrm{bw}$.

Group VI: Diabetic rats were submitted to supplementation with vitamin $\mathrm{D}_{3}$ (Cholecalciferol) (Sigma-Aldrich, St. Louis, MO,USA) dissolved in $0.3 \mathrm{~mL}$ olive oil at a dose of $12.5 \mu \mathrm{gkg}^{-1}$ bw and calcium gluconate gluconate (Acros Organic, New Jersey, USA; Purity $99 \%$ ) dissolved in distilled water at a dose of $100 \mathrm{mgkg}^{-1}$ bw.
Group VII: Diabetic rats received olive oil at a dose of $0.3 \mathrm{mLkg}^{-1} \mathrm{bw}$, serves as vehicle group.

Measurement of body weight: Body weight of each animal in control and experimental groups was measured and recorded immediately before diabetic induction and after 28 days of treatment.

Blood collection: Blood samples were collected via. retro-orbital puncture from all control and experimental animal groups with the help of microhematocrit tube at 4 weeks after administration. For serum preparation, blood samples were collected in Eppendorff's tubes and allowed to clot for $10 \mathrm{~min}$ by centrifuging, the serum was separated at $3000 \mathrm{rpm}$ for $10 \mathrm{~min}$ and stored in a refrigerator for the following procedures.

Estimation of serum biochemical parameters: Serum samples of all animal groups were used for the estimation of glucose, insulin, amylase and lipase levels at 28 days after treatment as follow.

Glucose level: Level of blood glucose was determined by glucose (Oxidase) Reagent Set. Catalogue number N6G51a-1L, obtained from TECO- Diagnostic, California USA. Glucose levels were expressed in $\mathrm{mgdL}^{-1}$.

Insulin level: Insulin was measured by a two-step sandwich chemiluminescent enzyme immunoassay method using immulite 2000 automated analyzer (Siemens healthcare diagnostics Inc., Deerfield, IL). Serum insulin levels were expressed in $\mu \mathrm{IUmL}^{-1}$.

Statistical analysis: Statistical analyses were performed by one way ANOVA test SPSS Software. The difference was considered significant at a value of $p<0.05$.

The effect of combined and individual supplementation of calcium and vitamin $\mathrm{D}_{3}$ on glycemic status, body weight, serum insulin levels and pancreatic enzyme activity were studied in diabetic rats.

Effect of different treatments on body weight: Significantly, body weight decreased in case of alloxanic diabetes during the experimental period. Changes in the body weight of normal and diabetic groups are shown in Table 1.

Neither treatment regimen could stop body weight loss accompanied by the diabetic state, however, the body weight loss varies with the treatment. Metformin was superior to other treatments in partially keeping body weight of diabetic rats, followed by vitamin $\mathrm{D}_{3}$, vitamin $\mathrm{D}_{3}$-calcium combination, calcium and finally, olive oil.

Effect of different treatments on serum glucose level: The sugar level of normal and treated animals were 
Res. J. Biol. Sci., 14 (3-6): 61-65, 2019

Table 1: Changes in the body weight (g) of normal and diabetic groups

\begin{tabular}{lll}
\hline Groups & Initial body weight & Final body weight \\
\hline Normal group & $261 \pm 4.23$ & $303.25 \pm 4.6$ \\
Diabetic group & $222.14 \pm 5.23$ & $173.6 \pm 13.5$ \\
Vitamin $\mathrm{D}_{3}$ group & $223.57 \pm 15.74$ & $207.9 \pm 9.9$ \\
Calcium group & $233.75 \pm 16.4$ & $210 \pm 20.4$ \\
Vitamin $\mathrm{D}_{3}+$ calcium group & $237.5 \pm 5.35$ & $213.75 \pm 5.2$ \\
Olive oil group & $236.25 \pm 4.98$ & $187.5 \pm 10.1$ \\
Metformin group & $236.48 \pm 5.2$ & $219.46 \pm 13.33$ \\
\hline
\end{tabular}

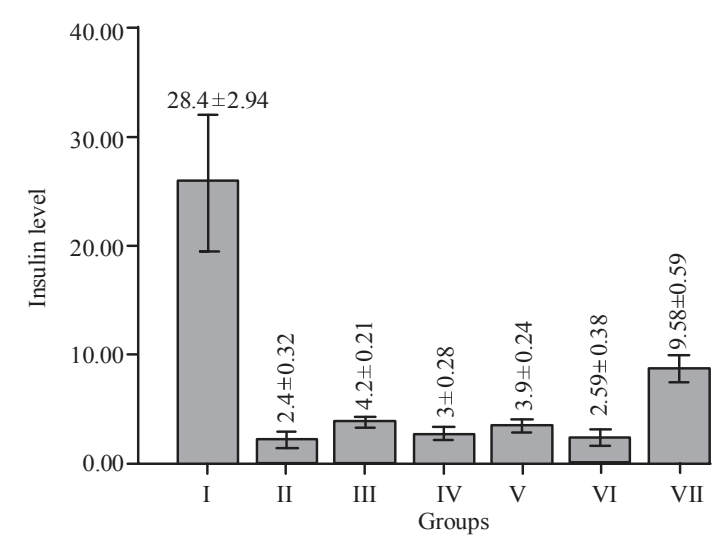

Fig. 1: Effect of determined types of treatments on serum glucose level after 28 days of experiment $(p<0.05)$. Group I: normal control, group II: diabetic control, group III: diabetic+vitamin $\mathrm{D}_{3}$, group IV: diabetic $+\mathrm{Ca}^{2+}$, group V: diabetic+vitamin $\mathrm{D}_{3}$ with $\mathrm{Ca}^{2+}$, group VI: diabetic+olive oil and group VII: diabetic + metformin

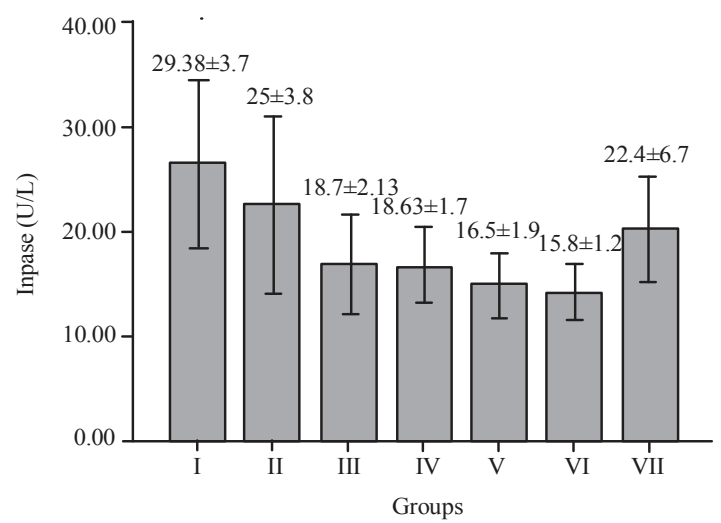

Fig. 2: Effect of determined types of treatments on serum insulin level after 28 days of experiment $(\mathrm{p}<0.05)$. Group I: normal control, group II: diabetic control, group III: diabetic+vitamin $\mathrm{D}_{3}$, group IV: diabetic $+\mathrm{Ca}^{2+}$, group $\mathrm{V}$ : diabetic + vitamin $\mathrm{D}_{3}$ with $\mathrm{Ca}^{2+}$, group VI: diabetic+olive oil and group VII: diabetic + metformin

measured at the end of the experiment as shown in Fig. 1. The mean of glucose level is highest in diabetic group, followed in sequence by olive oil, calcium, vitamin
$\mathrm{D}_{3}$-calcium combination, vitamin $\mathrm{D}_{3}$, metformin and normal. Metformin intake lowers serum glucose level in diabetic rats and it lowers it the most among tested groups.

Effect of different treatments on serum insulin level: Serum insulin level was measured for normal and treated animals at the end of the study as shown in Fig. 2. The serum insulin level is significantly decreased in alloxanic diabetic rats when compared to normal rats. Although, vitamin $\mathrm{D}_{3}$ and vitamin $\mathrm{D}_{3}$-calcium combination elevated insulin levels when given to diabetic rats but it is not statistically significant $(p<0.05)$. The level of insulin is not different among other groups when compared with diabetic rats. Neither calcium nor olive oil can increase insulin level in diabetic rats; metformin on the other hand, improves insulin level in diabetic rats.

\section{RESULTS AND DISCUSSION}

Biochemical parameters were analyzed resulted from the effect of vitamin $\mathrm{D}_{3}$ and calcium in alloxan induced-diabetic rats by recording blood glucose levels, serum insulin levels and serum levels of amylase and lipase.

Previous studies have recorded that diabetes is associated with vitamin D deficiency, reduces of calcium absorption in intestine, increase activity of parathyroid and bone loss, therefore, the levels of vitamin D and calcium is low in diabetic patients (Hamden et al., 2009; Phillip et al., 2013).

Cesar and colleagues 2006 proved that the individuals with type II diabetes had lower circulation levels of vitamin D than in type I diabetes (Di et al., 2006).

It is proposed that adequate calcium intake may improve the level of vitamin D which in turn can improve insulin level and lower glucose level. Taking these data together; vitamin D, calcium and vitamin D-calcium combination can ameliorate DM. We investigated these possibilities in this study.

Our result revealed that a general increase was noticed in the level of blood glucose in alloxan induced-diabetic rats. This increase is statistically significant compared to the normal group; vitamin $\mathrm{D}_{3}$ supplementation decreased the level of glucose. But it was $287 \pm 12.5 \mathrm{mgdL}^{-1}$ compared with the level in normal non diabetic rats $83.63 \pm 4.4 \mathrm{mgdL}^{-1}$. Calcium alone decreased glucose level but this was not statistically significant and its effect alone is less than when it is combined with vitamin $\mathrm{D}_{3}$.

Supplementation with vitamin $\mathrm{D}_{3}$ increases insulin level slightly and this increment is statistically not significant. Neither alone nor combined with calcium. While metformin increases insulin level which is statistically significant. 
Our results agree with the recent studies of Salum et al., (2012, 2013) who showed that supplementation of $12.5 \mathrm{\mu gkg}^{-1}$ cholecalciferol orally every day for 10 weeks to streptozotocin induced-diabetic rats reduction the blood glucose levels but it was not statically significant (Salum et al., 2012, 2013).

Our results were not in line with Santos and Vianna (2005) who examined the effect of cholecalciferol on blood glucose level for 14 days by gastric gavage in streptozotocin induced-diabetic rats, they recoded a significant decreased in blood glucose concentration as much as 40\%. Moreover, Anwar et al. (2013) who studied that administered cholecalciferol by dose $10 \mathrm{ng} 100 \mathrm{~g}^{-1}$ subcutaneously for 6 days significantly reduction as much as $26.31 \%$ of glucose levels.

It is evident that the difference in the duration of supplementation and route of administration of cholecalciferol and diabetes induction to experimental animals lead to a better glucose-lowering effect in these studies. Which the study of Rajesh concluded that intake of alloxan not only destroys the pancreatic $\beta$-cells but causes kidneys damage which is however reversible while streptozotocin selectively destroys pancreatic insulin secreting $\beta$-cells, causing diabetes close to T2DM in humans. In cause of cholecalciferol supplementation in alloxan induced-diabetic rats, may the kidneys damage that causes by alloxan effect on the second step activation of vitamin $\mathrm{D}_{3}$ (cholecalciferol) that take place in kidneys catalyzed by $1, \alpha$-hydroxylase which converts the $25(\mathrm{OH}) \mathrm{D}_{3}$ to the hormonally active metabolite, 1,25 dihydroxyvitamin $\mathrm{D}_{3}\left(1,25(\mathrm{OH})_{2} \mathrm{D}_{3}\right)$.

This finding is agree with the result of Hamden et al. (2009) who used the active form of vitamin $\mathrm{D}_{3}$ (5000 $\mathrm{IU} \mathrm{kg}^{-1}$ for 15 days by gastric gavage method) as supplement to alloxan induced-diabetic rats, this study indicated that the active form of vitamin $\mathrm{D}_{3}\left(1,25(\mathrm{OH})_{2} \mathrm{D}_{3}\right)$ can be effective in inhibiting hyperglycemia, oxidative stress and cell damage in pancreas, liver and kidneys by enhancing insulin level and sensitivity and antioxidant capacity (Hamden et al., 2009).

Pittas et al. (2007) evaluation the effect of the combined daily supplementation with $700 \mathrm{IU} \mathrm{d}^{-1}$ of vitamin $\mathrm{D}_{3}$ and $500 \mathrm{mg} \mathrm{d}^{-1}$ of calcium as calcium citrate for 3 years, supplementation attenuated the increase in fasting glycemia, only among those with glucose intolerance at baseline but had no effect among those with normal glucose tolerance.

Most of the cellular actions of vitamin D are mediated via the activation of VDR which is expressed and localized not only in the classical target tissues such as intestine, kidney and bone but also in many other tissues such as the pancreatic $\beta$ cell (Mathieu and Gysemans, 2006).
Results from previous studies provide evidence in support of the idea that vitamin D exerted its beneficial effect on $\beta$ cells by a particular activation of insulin turnover, accelerating the neoconversion of proinsulin to insulin. This hypothesis was supported by Burlon et al. (1999) by the complementary study of the $\left[{ }^{3} \mathrm{H}\right]$ tyrosin-labeled insulin/proinsulin in which vitamin $\mathrm{D}$ increases the ratio in a dose-dependent manner, showing that the newly synthesis insulin appeared more rapidly with a conversion rate exceeding that of islet from normal rats (Bourlon et al., 1999).

Our data indicated that metformin reduces the blood glucose levels in alloxan diabetic rats. It exerts its hypoglycemic effect by suppressing hepatic glucose production and increased peripheral tissue insulin sensitivity. This drug reduces gluconeogenesis in heaptocytes and amplifies glycogenesis and lipogenesis, reduces intestinal absorption of glucose and increases glucose intake in myocytes and adipocytes. The improvement in insulin sensitivity by metformin could be ascribed to its positive effects on insulin receptor expression and tyrosine kinase activity (Pournaghi et al., 2012).

Metformin has been shown to reduce free fatty acid oxidation by $10-30 \%$. Elevated levels of free fatty acid are commonly seen in DM and they contribute to increased hepatic glucose production and development of insulin resistance. Increased fatty acid oxidation inhibits key enzymes of the glycolytic pathway by accumulation of acetyl coenzyme A and citrate by-products of free fatty acid oxidation. Increased glucose 6-phosphate concentrations in turn, inhibit the hexokinase enzyme, resulting in reduced glucose uptake and oxidation. (Kirpichnikov et al., 2002; Cheng et al., 2006).

\section{CONCLUSION}

In conclusion, the data of this study revealed that the effect of vitamin $\mathrm{D}_{3}$ supplementation alone or combined with calcium on glucose level in alloxan induced diabetic rats was efficient more than supplementation of calcium alone but its effect on insulin level was not different compared with supplementation of calcium alone. In our experiment, metformin is by far the most hypoglycemic agent among tested treatments when compared with the effect of vitamin $\mathrm{D}_{3}$ supplementation with and without calcium.

\section{ACKNOWLEDGEMENT}

We thank the Al al-Bayt University, AlMafraq, Jordan for the support, also, we would like to thank the animal house unit staff of Faculty of Veterinary Medicine, Jordan University of Science and Technology, Jordan. 
Res. J. Biol. Sci., 14 (3-6): 61-65, 2019

\section{REFERENCES}

Afifi, F.U. and V. Kasabri, 2013. Pharmacological and phytochemical appraisal of selected medicinal plants from Jordan with claimed antidiabetic activities. Scientia Pharmaceutica, 81: 889-932.

Anwar, M.K., M.M. Hussain, M.A. Khan and T. Ahmad, 2013. Effect of cholecalciferol and levo carnitine on plasma glucose, plasma insulin and insulin resistance in type 2 diabetic rats. J. Pak. Med. Assoc. JPMA, 63: 374-379.

Bastaki, S., 2005. Diabetes mellitus and its treatment. Int. J. Diabetes Metab., 13: 111-134.

Bourlon, P.M., B. Billaudel and A. Faure-Dussert, 1999. Influence of vitamin D3 deficiency and 1, 25 dihydroxyvitamin $\mathrm{D} 3$ on de novo insulin biosynthesis in the islets of the rat endocrine pancreas. J. Endocrinol., 160: 87-95.

Cheng, J.T., C.C. Huang, I.M. Liu, T.F. Tzeng and C.J. Chang, 2006. Novel mechanism for plasma glucose-lowering action of metformin in streptozotocin-induced diabetic rats. Diabetes, 55: 819-825.

Di Cesar, D.J., R. Ploutz-Snyder, R.S. Weinstock and A.M. Moses, 2006. Vitamin D deficiency is more common in type 2 than in type 1 diabetes. Diabetes Care, 29: 174-174.

Hamden, K., S. Carreau, K. Jamoussi, S. Miladi and S. Lajmi et al., 2009. 1a, 25 dihydroxyvitamin D3: Therapeutic and preventiveNovember 22, 2019 effects against oxidative stress, hepatic, pancreatic and renal injury in alloxan-induced diabetes in rats. J. Nutr. Sci. Vitaminology, 55: 215-222.

Holick, M.F. and T.C. Chen, 2008. Vitamin D deficiency: A worldwide problem with health consequences. Am. J. Clin. Nutr., 87: 1080S-1086S.

Kirpichnikov, D., S.I. McFarlane and J.R. Sowers, 2002. Metformin: An update. Ann. Internal Med., 137: 25-33.

Kochupillai, N., 2008. The physiology of vitamin D: Current concepts. Indian J. Med. Res., 127: 256-262.

Kumar, R.S., B. Kar, N. Dolai, A. Bala and P.K. Haldar, 2012. Evaluation of antihyperglycemic and antioxidant properties of Streblus asper Lour against streptozotocin-induced diabetes in rats. Asian Pac. J. Trop. Dis., 2: 139-143.
Lips, P., 2012. Interaction between vitamin D and calcium. Scand. J. Clin. Lab. Invest., 72: 60-64.

Mathieu, C. and C. Gysemans, 2006. Vitamin D and diabetes. Diabetol, 22: 187-193.

Nikooyeh, B., T.R. Neyestani, M. Farvid, H. Alavi-Majd and A. Houshiarrad et al., 2011. Daily consumption of vitamin D-or vitamin $\mathrm{D}+$ calcium-fortified yogurt drink improved glycemic control in patients with type 2 diabetes: A randomized clinical trial. Am. J. Clin. Nutr., 93: 764-771.

Noda, M. and T. Mizoue, 2010. Relation between dietary calcium and vitamin D and risk of diabetes and cancer: A review and perspective. J. Clin. Metab. Diabetes JCMD, 1: 55-60.

Phillip, W., F. Service and B. Araud, 2013. Calcium homeostasis in diabetes mellitus. J. Clin. Endocrinol. Metaabolism, 49: 462-470.

Pittas, A.G., S.S. Harris, P.C. Stark and B. Dawson-Hughes, 2007. The effects of calcium and vitamin D supplementation on blood glucose and markers of inflammation in nondiabetic adults. Diabetes Care, 30: 980-986.

Pournaghi, P., R.A. Sadrkhanlou, S. Hasanzadeh and A. Foroughi, 2012. An investigation on body weights, blood glucose levels and pituitary-gonadal axis hormones in diabetic and metformin-treated diabetic female rats. Vet. Res. Forum, 3: 79-84.

Pravina, P., D. Sayaji and M. Avinash, 2013. Calcium and its role in human body. Int. J. Res. Pharm. Biomed. Sci., 4: 659-668.

Salum, E., J. Kals, P. Kampus, T. Salum and K. Zilmer et al., 2013. Vitamin D reduces deposition of advanced glycation end-products in the aortic wall and systemic oxidative stress in diabetic rats. Diabetes Res. Clin. Pract., 100: 243-249.

Salum, E., P. Kampus, M. Zilmer, J. Eha and M. Butlin et al., 2012. Effect of vitamin D on aortic remodeling in streptozotocin-induced diabetes. Cardiovasc. Diabetol., Vol. 11. 10.1186/1475-2840-11-58

Santos, R.D.S. and L.M. Vianna, 2005. Effect of cholecalciferol supplementation on blood glucose in an experimental model of type 2 diabetes mellitus in spontaneously hypertensive rats and Wistar rats. Clin. Chim. Acta, 358: 146-150.

Suganya, S., R. Narmadha, V.K. Gopalakrishnan and K. Devaki, 2012. Hypoglycemic effect of Costus pictus D. Don on alloxan induced type 2 diabetes mellitus in albino rats. Asian Pac. J. Trop. Dis., 2: 117-123. 